\title{
Malaria Elimination in China: Improving County-Level Malaria Personnel Knowledge of the 1-3-7 Strategy through Tabletop Exercises
}

\author{
Yuanyuan Cao, ${ }^{1}$ Chris Cotter, ${ }^{2}$ Weiming Wang, ${ }^{1}$ Yaobao Liu, ${ }^{1}$ Huayun Zhou, ${ }^{1}$ Guoding Zhu, ${ }^{1 *}$ and Jun Cao ${ }^{1,3,4 *}$ \\ ${ }^{1}$ National Health Commission Key Laboratory of Parasitic Disease Control and Prevention, Jiangsu Provincial Key Laboratory on Parasite and \\ Vector Control Technology, Jiangsu Institute of Parasitic Diseases, Wuxi, People's Republic of China; ${ }^{2}$ Malaria Elimination Initiative, Global Health \\ Group, University of California, San Francisco, San Francisco, California; ${ }^{3}$ Center for Global Health, School of Public Health, Nanjing Medical \\ University, Nanjing, People's Republic of China; ${ }^{4}$ Public Health Research Center, Jiangnan University, Wuxi, People's Republic of China
}

\begin{abstract}
As China moves to the prevention of reestablishment of malaria, maintaining skills for malaria in county personnel on the "1-3-7" surveillance and response strategy is critical. China's "1-3-7" strategy defines targets used to guide and monitor malaria case reporting, investigation, and response, respectively: reporting of malaria cases within 1 day, their confirmation and investigation within 3 days, and the appropriate public health response to prevent further transmission within 7 days. Assessing the knowledge of local CDC malaria personnel on the "1-3-7" surveillance and response strategy is urgently needed. In June 2016, two different training modules (classroom-style teaching and tabletop exercises) were conducted for 125 CDC staff in Jiangsu Province, China, to determine the effectiveness of the two training modules on CDC staff knowledge and learning of the "1-3-7" strategy. The classroom-style training module just imparted the malaria knowledge to participants through teaching. Tabletop exercises were carried out through discussion-based scenarios using questions and answers on the "1-3-7" strategy. Questionnaires assessing knowledge improvement were designed and administered to personnel responsible for malaria surveillance and response activities, including at baseline and end line. Overall, knowledge of the "1-3-7" strategy for malaria elimination was $63.2 \%$ correct at baseline, $70.6 \%$ after implementing a classroom-style teaching module $\left(X^{2}=11.20, P=0.001\right)$, and $84.6 \%$ after the tabletop exercise module $\left(X^{2}=48.82, P<0.001\right)$. The knowledge of each component of the "1-3-7" strategy improved significantly after the tabletop exercise module. The total proportion of respondents with a high score (greater than or equal to $75 \%$ ) was $82.7 \%$ in the classroom-style module and $95.2 \%$ in the tabletop exercise module. The proportion of respondents with a high score significantly increased after tabletop exercises in the stratified demographic groups of men who work at the county CDC level, have a bachelor's degree, hold a professional title as professor or assistant, are aged 31-50 years, and have attained 11-20 years of service with the CDC compared with the classroom-style module. Acceptability of the classroom-style module $(78.2 \%)$ compared with tabletop exercises (94.4\%) by the CDC malaria personnel increased significantly $\left(x^{2}=11.96, P=0.004\right)$. Feedback from participants on the modules suggest the tabletop exercises were an effective training method, which could maintain and improve the knowledge and capacity for malaria surveillance and response in basic CDC level personnel in China.
\end{abstract}

\section{INTRODUCTION}

An estimated 3.7 billion people in more than 90 countries and territories are at risk of malaria globally, with an estimated 219 million malaria cases and approximately 435,000 deaths occurring in 2017. ${ }^{1}$ Historically, malaria has been one of the most important infectious diseases in China. Wide-scale efforts on malaria prevention and control since 1956 have reduced reported malaria cases from 6.97 million to 14,000 across China by $2009 .^{2}$ Among 24 malaria-endemic provinces in China, only 63 counties $(2.89 \%)$ had malaria incidence rates of more than 1 case per 10,000 population in $2009 .{ }^{3}$ Given this progress in malaria control, China launched its National Malaria Elimination Action Plan (NMEAP) in 2010 with the goal of elimination by $2020 .^{4}$

To support the goal of the NMEAP, China's "1-3-7" surveillance and response strategy was developed and scaled up to a total 31 provinces in China in early 2012. The "1-3-7" approach defines targets used to guide and monitor malaria case reporting, case investigation, and follow-up response, respectively: reporting of malaria cases within 1 day through China's web-based health information system by the local healthcare provider; case confirmation and investigation

\footnotetext{
*Address correspondence to Guoding Zhu or Jun Cao, Jiangsu Institute of Parasitic Diseases, Meiyuan Yangxiang 117, Wuxi, Jiangsu Province 214064, People's Republic of China. E-mails: jipdzhu@
} hotmail.com or caojuncn@hotmail.com within 3 days by the China county-level CDC where the case is reported, to determine where the case originated (local or imported); and the appropriate public health response within 7 days by the China county-level CDC where the patient resides and/or works to prevent further transmission. ${ }^{5,6}$ Since national rollout of China's "1-3-7" strategy, time-bound targets for case reporting, case investigation, and foci response have been achieved in a large and diverse country such as China. ${ }^{7}$ The "1-3-7" strategy has been incorporated as a surveillance and response strategy within the Greater Mekong Subregion, including Cambodia and Thailand. ${ }^{8}$

Malaria personnel at the prefecture and county CDC are the frontline implementers of "1-3-7" activities and are involved in malaria case diagnosis and reporting, case confirmation, and epidemiological investigation, in addition to conducting specific control measures for foci disposal in the community. It is important that the CDC malaria personnel have an accurate understanding of how to implement the "1-3-7" strategy to ensure an effective response to malaria cases and quickly reduce any opportunities for secondary transmission arising from imported malaria.

Recent studies have highlighted the knowledge and performance of malaria personnel conducting "1-3-7" strategy activities. $^{7,9}$ Questionnaires administered to malaria personnel on their knowledge of the standard operating procedures (SOPs) have shown that respondents did not understand the SOPs for reactive case detection (RACD). ${ }^{10}$ Furthermore, the management of imported malaria cases will remain a major 
challenge for achieving and sustaining malaria elimination in China. ${ }^{7}$ The aim of this study was to evaluate and compare the effects of different types of training modules (classroom-style or tabletop exercises) given to county-level CDC malaria personnel in Jiangsu Province on the knowledge of the "1-3-7" surveillance and response strategy.

\section{METHODS}

Study site. Jiangsu Province is located in the southeast of China. The province covers an area of 102.6 thousand square kilometers, $1.06 \%$ of the total area of China. Situated in a transition belt from a subtropical to temperate zone, Jiangsu Province has a typical monsoon climate. Anopheles sinensis and Anopheles anthropophagus are the main mosquito vectors, and the climate and environment is suitable for the breeding of Anopheles mosquitoes. ${ }^{11}$ There are a total of 13 prefectural cities and 100 counties in Jiangsu Province.

Study participants. A total of 125 participants were selected from all 13 prefecture CDCs and 100 county CDCs across Jiangsu Province. One to two government personnel in each county and prefecture CDC are responsible for the daily management of malaria control and other parasites.

Study design. All surveys and training modules were carried out in June 2016 at the Jiangsu Institute of Parasitic Diseases (JIPD), an international training center in Wuxi, Jiangsu. CDC malaria personnel took part in the malaria training module (using classroom-style teaching) and tabletop exercises (using discussion-based sessions) with pre- and postintervention surveys (Figure 1). For the classroom-style teaching course, this module only imparted malaria knowledge to all participants, including China's "1-3-7" surveillance and response strategy, such as malaria diagnosis and reporting, epidemiological survey and malaria case verification, RACD, foci investigation and disposal, and the progress of malaria elimination in Jiangsu.

Next, tabletop exercises were carried out using questions and answers through "1-3-7" strategy malaria disposal scenarios for all participants. During the tabletop exercises, all participants were randomly assigned to 13 different groups based on different scenarios of malaria diagnosis and reporting, sample collection, case confirmation, RACD, foci investigation, and malaria risk identification. Then, a facilitator presented questions to all participants on the "1-3-7" strategy based on particular scenarios. Randomly selected groups then answered questions and were evaluated by a different randomly selected group. For example, answers were provided by random group $A$, and then random group $B$ would assess the above answers. After this process, reference answers were presented to all participants, and each group openly discussed through the guides of the facilitator. Finally, JIPD experts evaluated and commented on each group's answers. Tabletop exercises allow participants to dispose and respond to scenarios by performing their assigned duties in a simulated setting (Figure 1). For both training modules, each key segment of malaria foci disposal in China's "1-3-7" surveillance and response strategy was covered. After the tabletop exercises, a questionnaire assessed the knowledge of all study participants post-training.

Questionnaires. Three different standardized questionnaires were designed by the JIPD malaria division based on previously published descriptions of China's "1-3-7" surveillance and response strategy. 5,6 Questionnaires captured participant sociodemographic characteristics (age, gender, service year, CDC level, education, and professional title) and contained eight multiple-choice questions including the following: 1) knowledge of the "1-3-7" work activities, 2) differences between malaria control and elimination stages, 3 ) "1-3-7" strategy definition, 4) description of "1-3-7" strategy, 5) responsible units for malaria case confirmation and epidemiological investigation, 6) assessment criteria for malaria infection source, 7) key points of foci investigation, and 8) foci transmission risk identification.

A standardized questionnaire was given to each participant at the end of each intervention to evaluate malaria personnel knowledge. Acceptability of each intervention was evaluated in a post-intervention questionnaire. Degree of training module acceptability was divided into three levels: 3) satisfied, 2) fair, and 1) needs improvement.

Data extraction and quality control. At the beginning of each module, a baseline survey was conducted with all participants. After the classroom-style and tabletop exercise modules, questionnaires were administered to all study participants to

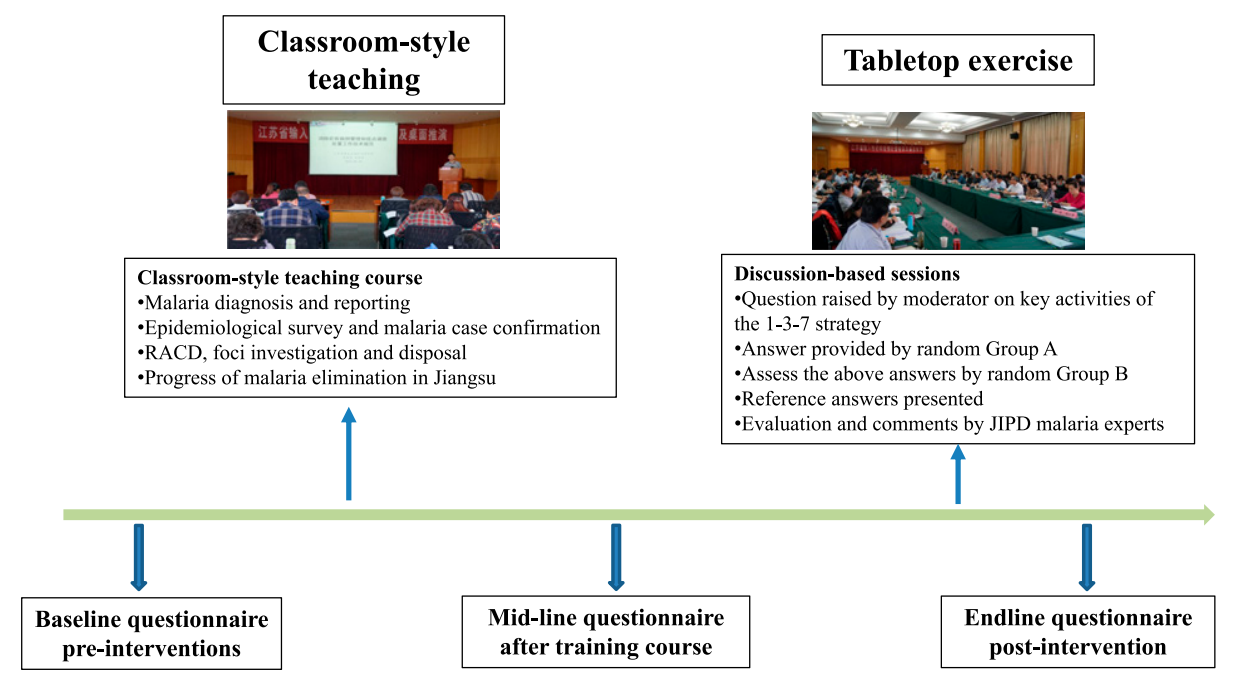

FigurE 1. Flowchart of surveys and different training modules. This figure appears in color at www.ajtmh.org. 
determine their knowledge of "1-3-7" activities. Study participants completed their post-training evaluations in the JIPD training center. A database of the questionnaire responses was created in Epidata version 3.1 (the EpiData Association, Odense, Denmark). Data were independently double entered by two JIPD staff members. If discrepancies in data entry were identified, corrections were made by an independent third JIPD staff member by reviewing the questionnaire data.

Data analysis. The datasets included basic characteristics of all participants and were input into Excel (Microsoft Office 2007, Microsoft Corp., Redmond, WA). The study population was stratified and analyzed by the following: gender, CDC level, education, professional title, age-group, and service years. Categorical variables such as knowledge of the "1-3-7" strategy and degree of evaluation acceptability were carried out by chi-square $\left(x^{2}\right)$ tests. The likelihood-ratio correction was used when the expected frequency in any cell was five or less. Knowledge (as a \%) equaled the number of correct answers to relevant knowledge questions divided by the total number of answers to relevant knowledge questions multiplied by 100 . A high score (greater than or equal to $75 \%$ ) meant that each respondent gave six correct answers of eight multiplechoice questions in each questionnaire. $P$-values less than 0.05 were considered statistically significant. All statistical analyses were performed in STATA version 12 (College Station, TX).

Ethics approval. All respondent identifiers were removed from the data before extraction from the database. Nonpersonal identifiers were used during analysis and presentation. Informed written consent was obtained from all participants before trainings. The study was approved by the Institutional Review Board of JIPD (IRB00004221), Wuxi, China.

\section{RESULTS}

Demographic characteristics of the study participants. Of the 125 participants targeted, 121 responded to the baseline survey, 110 to the classroom-style questionnaire, and 107 to the tabletop exercise questionnaire. Of the 121 baseline survey questionnaires obtained, $56.2 \%(68 / 121)$ of the respondents were male and $40.5 \%$ (49/121) female, with four nonresponses (4/121, 3.3\%). For the malaria CDC staff, 69.4\% (84/121) of personnel worked at the county level. For educational background, $61.1 \%(71 / 121)$ had a bachelor's degree and $19.8 \%$ (24/121) had an associate's degree, accounting for $80.9 \%$ (98/ 121) overall; $61.1 \%(74 / 121)$ of respondents held assistant- or primary-level job titles, and 25.6\% (31/121) were professors. All of the respondents were aged between 21 and 60 years, with the 31- to 40 -year-old group accounting for $28.1 \%$ (34/121) and the 41 - to 50 -year-old group accounting for $33.4 \%$ (41/121). For the number of service years, $70.3 \%$ (79/121) of respondents worked on malaria control for more than 10 years (Table 1 ).

Respondent knowledge of the "1-3-7" strategy. In the baseline survey, $58.7 \%(71 / 121)$ of the participants noted that they understood the "1-3-7" strategy and $51.2 \%(62 / 121)$ could distinguish differences between malaria control and elimination stages (Table 1); 39.7\% (48/121) understood the "1-3-7" strategy description, and 35.5\% (43/121) could draw a clear distinction of identifying foci transmission risks. Differences were identified on the knowledge of all respondents for China's "1-3-7" strategy at baseline survey (63.2\%), after the classroom-style training module (70.6\%), and after tabletop exercises (84.6\%) (Figure 2).

Questionnaire results were divided into seven parts for further analysis. Total knowledge of all respondents of the "13-7" strategy increased from $63.2 \%$ at baseline to $70.6 \%$ after the classroom-style training module $\left(x^{2}=11.20, P=0.001\right)$, and $84.6 \%$ after the tabletop exercises $\left(X^{2}=105.73, P<0.001\right)$ (Table 2). Compared with results at baseline (51.2\%), respondents could identify the differences between malaria control and malaria elimination stages after the classroomstyle training module $\left(67.3 \% ; \mathrm{x}^{2}=6.12, P=0.013\right)$ and tabletop exercises (79.4\%; $x^{2}=19.71, P<0.001$ ); $94.2 \%$ of respondents knew China's "1-3-7" strategy definition when surveyed at baseline, $92.7 \%$ after the classroom-style training

TABLE 1

Demographic characteristics of CDC malaria personnel from questionnaires after "1-3-7" strategy baseline survey

\begin{tabular}{|c|c|c|c|}
\hline Item & Characteristics & $N$ & Proportion (\%) \\
\hline \multirow[t]{2}{*}{ Gender* } & Male & 68 & 56.2 \\
\hline & Female & 49 & 40.4 \\
\hline \multirow[t]{2}{*}{ CDC level* $^{*}$} & Prefecture & 27 & 22.3 \\
\hline & County & 84 & 69.4 \\
\hline \multirow[t]{4}{*}{ Education* } & Master & 11 & 9.1 \\
\hline & Bachelor & 74 & 61.1 \\
\hline & Associate degree & 24 & 19.8 \\
\hline & High-school degree & 7 & 5.8 \\
\hline \multirow[t]{3}{*}{ Professional title* } & Professor & 31 & 25.6 \\
\hline & Assistant & 47 & 38.8 \\
\hline & Primary & 27 & 22.3 \\
\hline \multirow[t]{4}{*}{ Age-group (years)* } & $21-30$ & 19 & 15.7 \\
\hline & $31-40$ & 34 & 28.1 \\
\hline & $41-50$ & 41 & 33.4 \\
\hline & $51-60$ & 22 & 18.2 \\
\hline \multirow[t]{4}{*}{ Service years ${ }^{\star}$} & $1-10$ & 36 & 29.7 \\
\hline & $11-20$ & 21 & 17.3 \\
\hline & $21-30$ & 39 & 32.2 \\
\hline & $31-40$ & 19 & 15.7 \\
\hline \multirow[t]{4}{*}{ Whether understood "1-3-7" strategy } & Very & 71 & 58.7 \\
\hline & A little & 38 & 31.4 \\
\hline & Not so much & 8 & 6.6 \\
\hline & Little & 4 & 3.3 \\
\hline
\end{tabular}

${ }^{\star}$ Questionnaire data partially missed because of respondent not providing a response. 


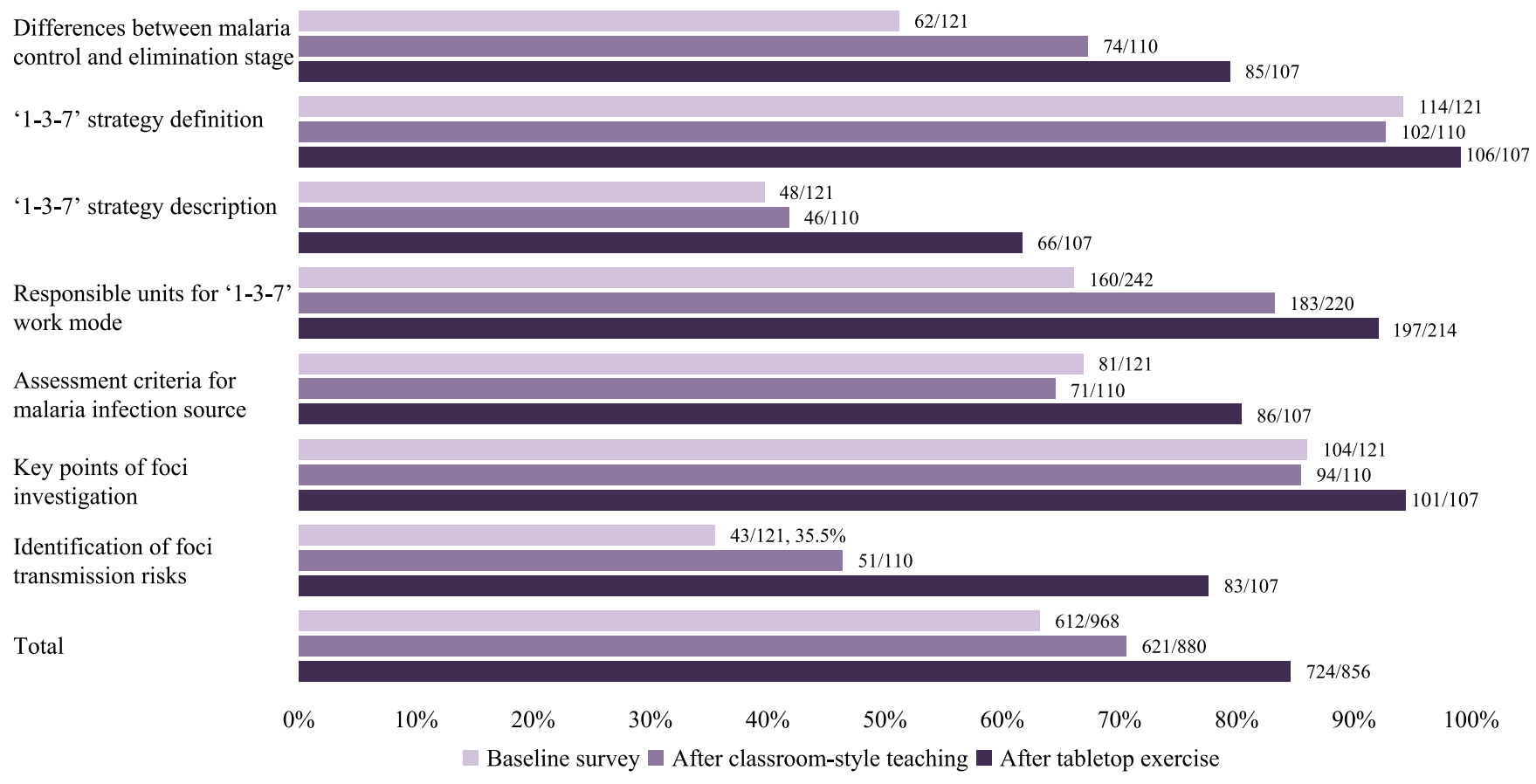

FIGURE 2. Knowledge of each aspect for China's "1-3-7" surveillance and response strategy among baseline survey, after classroom-style teaching, and after tabletop exercises. This figure appears in color at www.ajtmh.org.

module, and $99.1 \%$ after tabletop exercises $\left(x^{2}=5.48, P=\right.$ $0.019)$. There were no significant differences on whether respondents understood the "1-3-7" strategy description between baseline survey (39.7\%) and classroom-style training module $\left(41.8 \% ; x^{2}=0.11, P=0.74\right)$; however, after the tabletop exercises, respondents' knowledge of the strategy description increased from $41.8 \%$ to $61.7 \%\left(\mathrm{x}^{2}=8.57, P=\right.$ 0.003).

Units responsible for the epidemiological survey and malaria case confirmation answered correctly after the classroom-style training module $(83.2 \%)$ and tabletop exercises $\left(92.1 \% ; X^{2}=\right.$ $\left.17.55, P<0.001 ; x^{2}=7.84, P=0.005\right)$. There were no differences between baseline survey (66.9\%) and classroom-style training module $\left(64.5 \% ; x^{2}=0.15, P=0.701\right)$ for malaria infection source criteria, but knowledge significantly increased after tabletop exercises $\left(80.4 \% ; X^{2}=6.79, P=0.009\right) ; 85 \%$ of respondents knew key points of foci investigation between the baseline survey and the two modules. Respondents' knowledge on the identification of foci transmission risks increased to
$77.6 \%$ after tabletop exercises from $46.4 \%$ at baseline $\left(x^{2}=\right.$ 22.36, $P<0.001$ ) (Figure 2, Table 2).

Stratified univariate analysis between the classroom-style training module and tabletop exercises by different demographic and other characteristics is shown in Table 3. The total proportion of respondents with a high score was $82.7 \%$ in the classroom-style training module and $95.2 \%$ in tabletop exercises. The proportion of respondents with a high score significantly increased after tabletop exercises in the stratified groups for men who work at the county CDC level, have a bachelor's degree, hold a professional title as professor or assistant, are of 31-50 years of age, and have attained 11-20 years of service with the CDC (Table 3) compared with the classroom-style training module.

Evaluation of classroom-style training module and tabletop exercise acceptability. An evaluation of training participant acceptability was conducted to estimate the degree to which the classroom-style training and tabletop exercises may support their work activities. The degree of acceptability of the

TABLE 2

Comparison of knowledge among baseline survey, classroom-style module, and tabletop exercises

\begin{tabular}{|c|c|c|c|c|}
\hline \multirow[b]{2}{*}{ Items } & \multicolumn{2}{|c|}{$\begin{array}{l}\text { Comparison of baseline survey and after } \\
\text { classroom-style module }\end{array}$} & \multicolumn{2}{|c|}{$\begin{array}{l}\text { Comparison of classroom-style module } \\
\text { and after tabletop exercises }\end{array}$} \\
\hline & $x^{2}$ & $P$-value & $x^{2}$ & $P$-value \\
\hline $\begin{array}{l}\text { Differences between malaria control and } \\
\text { elimination stage }\end{array}$ & 6.12 & 0.013 & 4.1 & 0.043 \\
\hline "1-3-7" strategy definition & 0.21 & 0.647 & 5.48 & 0.019 \\
\hline “1-3-7" strategy description & 0.11 & 0.74 & 8.57 & 0.003 \\
\hline Responsible units for "1-3-7" strategy & 17.55 & 0.000 & 7.84 & 0.005 \\
\hline $\begin{array}{l}\text { Assessment criteria for malaria infection } \\
\text { source }\end{array}$ & 0.15 & 0.701 & 6.79 & 0.009 \\
\hline Key points of foci investigation & 0.01 & 0.914 & 4.76 & 0.029 \\
\hline Identification of foci transmission risks & 2.80 & 0.094 & 22.36 & 0.000 \\
\hline Total & 11.20 & 0.001 & 48.82 & 0.000 \\
\hline
\end{tabular}


TABLE 3

Univariate analysis of knowledge of China's "1-3-7" strategy knowledge between classroom-style module and tabletop exercises

\begin{tabular}{|c|c|c|c|c|c|c|c|}
\hline \multirow[b]{2}{*}{ Items } & \multicolumn{3}{|c|}{ Classroom-style module } & \multicolumn{4}{|c|}{ Tabletop exercises } \\
\hline & Stratified groups & $N$ & No. of high score (\%) & $N$ & No. of high score (\%) & $x^{2}$ & $P$-value \\
\hline \multirow[t]{2}{*}{ Gender } & Male & 55 & $44(80)$ & 54 & $51(94.4)$ & 5.37 & 0.020 \\
\hline & Female & 47 & $40(85.1)$ & 47 & $45(95.7)$ & 3.24 & 0.072 \\
\hline \multirow[t]{2}{*}{ CDC level } & Prefecture & 19 & 17 (89.5) & 24 & $24(100.0)$ & 3.39 & 0.066 \\
\hline & County & 82 & $66(80.5)$ & 77 & 72 (93.5) & 5.87 & 0.015 \\
\hline \multirow[t]{4}{*}{ Education } & Master & 9 & 9 (100.0) & 8 & 8 (100.0) & NA & NA \\
\hline & Bachelor & 71 & $57(80.3)$ & 73 & 71 (97.3) & 11.63 & 0.001 \\
\hline & Associate degree & 22 & 20 (90.9) & 22 & $19(86.4)$ & 0.23 & 0.634 \\
\hline & High school degree & 4 & $3(75.0)$ & 1 & $1(100)$ & 0.51 & 0.477 \\
\hline \multirow[t]{3}{*}{ Professional title } & Professor & 26 & $23(88.5)$ & 30 & $30(100)$ & 4.8 & 0.028 \\
\hline & Assistant & 49 & 38 (77.6) & 44 & 41 (93.2) & 4.7 & 0.030 \\
\hline & Primary & 31 & $27(87.1)$ & 21 & 20 (95.2) & 1.04 & 0.308 \\
\hline \multirow[t]{4}{*}{ Age-group } & $21-30$ & 18 & 16 (88.9) & 16 & 14 (87.5) & 0.02 & 0.900 \\
\hline & $31-40$ & 35 & 28 (80.0) & 32 & 31 (96.9) & 5.08 & 0.024 \\
\hline & $41-50$ & 33 & 26 (78.8) & 34 & $34(100.0)$ & 10.76 & 0.001 \\
\hline & $51-60$ & 18 & 16 (88.9) & 22 & 20 (90.9) & 0.05 & 0.833 \\
\hline \multirow[t]{4}{*}{ Service year } & $1-10$ & 36 & 32 (88.9) & 33 & 30 (90.9) & 0.08 & 0.781 \\
\hline & $11-20$ & 21 & 16 (76.2) & 22 & $22(100.0)$ & 7.86 & 0.005 \\
\hline & 21-30 & 32 & 25 (78.1) & 31 & $29(93.5)$ & 3.22 & 0.073 \\
\hline & $31-40$ & 16 & 14 (82.9) & 17 & 17 (100) & 3.03 & 0.082 \\
\hline Total & & 624 & $516(82.7)$ & 608 & 579 (95.2) & 48.98 & 0.000 \\
\hline
\end{tabular}

classroom-style malaria training (78.2\%) and tabletop exercises $(94.4 \%)$ increased $\left(X^{2}=11.96, P=0.004\right)$. For whether the intervention topics were related to their actual work, respondents noted the classroom-style training (88.2\%) and tabletop exercises $(96.3 \%)$ were very relevant for malaria management and foci disposals. Most respondents (82.7\% and 91.6\%) thought that content settings of two interventions were reasonable. Respondents were satisfied with the forms associated with the classroom-style module (84.5\%) and tabletop exercise (94.4\%) training; $90.7 \%$ of the respondents believed that the tabletop exercises achieved the expected level of knowledge of "1-3-7" activities (Table 4).

\section{DISCUSSION}

This study demonstrated that the overall knowledge of the "13-7" strategy for basic CDC malaria personnel was relatively low; however, tabletop exercises were an effective intervention for malaria personnel to improve their understanding of the strategy compared with classroom-style training. Previous studies have shown that tabletop exercises on emergency preparedness are an innovative method that can be used to assess and improve public health emergency response capabilities. ${ }^{12,13}$

The "1-3-7" strategy has played an important role since the beginning of the NMEAP in efficiently detecting, treating, and responding to individual malaria cases, and promptly eliminating the source of infection. ${ }^{14,15}$ The strategy has been well executed even in the most difficult border areas between Myanmar and China, although a need to further strengthen the "3" indicator in the 18 border counties of Yunnan Province has been noted. ${ }^{16}$ The "1-3-7" malaria surveillance and response strategy rolled out nationally in early $2012,{ }^{5}$ yet weaknesses in the understanding of the strategy and implementation by the basic CDC malaria personnel remain. Concerns have been raised about a lack of understanding of strategic concepts and objectives in the malaria elimination stages. ${ }^{17}$ Results demonstrated here, and identified previously, ${ }^{7,9}$ highlight that most study respondents had a low understanding of the "1-37" strategy work activities. In this study, through a classroomstyle training module, simple questions on the differences between malaria control and elimination stages, and identifying the responsible units for the "1-3-7" activities, improved knowledge of the strategy. However, this study was unable to identify differences between baseline survey and classroomstyle training for other key strategy components, such as the assessment criteria for malaria case origination, foci investigation, and identification of foci transmission risks. Although basic knowledge of the strategy could be improved through the classroom-style training module, overall knowledge improvement was low. Tabletop exercises improved the knowledge of each aspect on China's "1-3-7" surveillance and response strategy.

TABLE 4

Evaluation of acceptability degree for the classroom-style module and tabletop exercises

\begin{tabular}{|c|c|c|c|c|c|c|c|c|}
\hline \multirow[b]{2}{*}{ Items } & \multicolumn{4}{|c|}{ Classroom-style } & \multicolumn{4}{|c|}{ Tabletop exercises } \\
\hline & N & Satisfied (\%) & Fair (\%) & Needs improvement (\%) & N & Satisfied (\%) & Fair (\%) & Needs improvement (\%) \\
\hline Whether related with your actual work & 110 & $97(88.2)$ & $12(10.9)$ & $1(0.9)$ & 107 & 103 (96.3) & $4(3.7)$ & $0(0)$ \\
\hline $\begin{array}{l}\text { Whether content was reasonable for the } \\
\text { work setting }\end{array}$ & 110 & $91(82.7)$ & 19 (17.3) & $0(0)$ & 107 & 98 (91.6) & $9(8.4)$ & $0(0)$ \\
\hline Whether satisfied with training forms & 110 & $93(84.5)$ & $17(15.5)$ & $0(0)$ & 107 & $101(94.4)$ & $6(5.6)$ & $0(0)$ \\
\hline $\begin{array}{l}\text { Whether expected to achieve effect on the } \\
\text { "1-3-7" strategy }\end{array}$ & 110 & $84(76.4)$ & $22(20)$ & $4(3.6)$ & 107 & $97(90.7)$ & $10(9.3)$ & $0(0)$ \\
\hline Total acceptability degree & 110 & $86(78.2)$ & $22(20)$ & $2(1.8)$ & 107 & $101(94.4)$ & $6(5.6)$ & $0(0)$ \\
\hline
\end{tabular}


A continued challenge for malaria elimination in China remains imported malaria. ${ }^{18}$ In 2016 and 2107, there were 3,321 and 2,816 imported malaria cases in China, ${ }^{19,20}$ posing a threat to achieving and sustaining malaria elimination in China. Many malariareceptive areas remain in China, especially during the warm summer months. Continued importation of malaria across its southern border with Myanmar, and from Chinese workers returning from overseas, ${ }^{21}$ is a threat to maintaining the interruption of indigenous malaria transmission in China. It is important that CDC malaria personnel can recognize and appropriately respond to imported malaria to prevent malaria from reestablishing. Tabletop exercises can be used to train local CDC malaria personnel throughout the year in high-risk areas and during potential malaria-related outbreak emergencies in China.

Study results also identified that the respondents who were 31 - to 50-year-old men, worked at the county CDC level, were educated, and had 11-20 years of work service more easily acquired an improved understanding of the "1-3-7" strategy after the tabletop exercises. The respondents with these characteristics had a lower understanding than other groups after the classroom-style training module; however, the total proportion of these respondents with a high score was much greater than that in the baseline survey.

Both the classroom-style training module and tabletop exercises improved understanding of the "1-3-7" malaria surveillance and response strategy. Results increased significantly after tabletop exercises compared with the classroomstyle module; $91.6 \%$ and $94.4 \%$ of basic CDC malaria personnel provided positive feedback for the content and forms on tabletop exercises, and concluded it was relevant with their work on the "1-3-7" strategy. For post-elimination, basic CDC malaria personnel will need to be equipped with the knowledge of how to implement the "1-3-7" strategy. The use of tabletop exercises at regular training intervals may play a role in maintaining the necessary skills in malaria surveillance and response in Jiangsu Province and China where potential malaria foci exist.

This study has limitations in that CDC malaria personnel of Jiangsu Province participated and may not represent the same knowledge of malaria personnel in other provinces in China. Tabletop exercises are a novel training tool for local CDC malaria personnel, yet it is possible that the questions may have been misunderstood. Although the tabletop exercises demonstrated greater knowledge gain after completion, and therefore potentially a better learning strategy, study authors did not randomize the groups to either classroom-style training module or the tabletop exercises. Therefore, it is impossible from this study to know the true effect of one training style alone.

\section{CONCLUSION}

The knowledge of the "1-3-7" strategy for basic CDC personnel was low overall. More interactive and discussionbased methods, such as the tabletop exercises, can increase the knowledge of basic CDC malaria personnel on China's "1-3-7" surveillance and response strategy. Maintaining the knowledge and capacity of local malaria personnel will be critical as China works toward its goal of malaria elimination in 2020 and to maintain that achievement thereafter.

Received July 26, 2019. Accepted for publication January 9, 2020.

Published online February 24, 2020.

Note: Supplemental information appears at www.ajtmh.org.
Acknowledgments: We thank the cooperation of malaria personnel of local CDC in all 13 prefectures and 100 counties of Jiangsu Province for the training.

Financial support: This study was supported by the Jiangsu Provincial Department of Science and Technology (No. BM2018020), the Jiangsu Provincial Key Research and Development Program (No. BE2016631), the Jiangsu Provincial Project of Invigorating Health Care through Science, Technology, and Education (ZDXKA2016016), and the Jiangsu Provincial Health Commission (H2017029, H2018102, and X201828).

Authors' addresses: Yuanyuan Cao, Weiming Wang, Yaobao Liu, Huayun Zhou, Guoding Zhu, and Jun Cao, National Health Commission Key Laboratory of Parasitic Disease Control and Prevention, Jiangsu Provincial Key Laboratory on Parasite and Vector Control Technology, Jiangsu Institute of Parasitic Diseases, Wuxi, People's Republic of China, E-mails: caopepsi@163.com, jipd@163.com, yaobao0721@163.com, hyzhou88@163.com, jipdzhu@hotmail.com, and caojuncn@hotmail.com. Chris Cotter, Malaria Elimination Initiative, Global Health Group, University of California, San Francisco, San Francisco, CA, E-mail: chris.cotter@ucsf.edu.

\section{REFERENCES}

1. World Health Organization, 2018. World Malaria Report 2018. Geneva, Switzerland: WHO, 6.

2. Tang LH, 1999. Achievements of malaria control and research in China [in Chinese]. Chin J Parasitol Parasitic Dis 17: 257-259.

3. Zhou SS, Wang Y, Xia ZG, 2011. Malaria situation in the People's Republic of China in 2009 [in Chinese]. Chin J Parasitol Parasitic Dis 29: 1-3.

4. Lei ZL, Wang LY, 2012. Control situation and primary task of key parasitic diseases in China [in Chinese]. Chin J Parasitol Parasitic Dis 30: 1-5.

5. Cao J, Sturrock HJ, Cotter C, Zhou S, Zhou H, Liu Y, Tang L, Gosling RD, Feachem RG, Gao Q, 2014. Communicating and monitoring surveillance and response activities for malaria elimination: China's "1-3-7" strategy. PLoS Med 11: e1001642.

6. Cao J, Zhou SS, Zhou HY, Yu YB, Tang LH, Gao Q, 2013. Malaria from control to elimination in China: transition of goal, strategy and interventions [in Chinese]. Chin J Schistosomiasis Control 25: 439-443.

7. Zhou SS et al., 2015. China's 1-3-7 surveillance and response strategy for malaria elimination: is case reporting, investigation and foci response happening according to plan? Infect Dis Poverty 4: 55.

8. World Health Organization, 2016. Strategy for Malaria Elimination in the Greater Mekong Subregion (2015-2030). Geneva, Switzerland: WHO, 17.

9. Lu G, Liu Y, Beiersmann C, Feng Y, Cao J, Muller O, 2016. Challenges in and lessons learned during the implementation of the 1-3-7 malaria surveillance and response strategy in China: a qualitative study. Infect Dis Poverty 5: 94.

10. Cotter C, Sudathip P, Herdiana H, Cao Y, Liu Y, Luo A, Ranasinghe N, Bennett A, Cao J, Gosling RD, 2017. Piloting a programme tool to evaluate malaria case investigation and reactive case detection activities: results from 3 settings in the Asia Pacific. Malar J 16: 347.

11. Zhu $\mathrm{G}$ et al., 2014. The colonization of pyrethroid resistant strain from wild Anopheles sinensis, the major Asian malaria vector. Parasites Vectors 7: 582

12. Savoia E, Testa MA, Biddinger PD, Cadigan RO, Koh $\mathrm{H}$, Campbell P, Stoto MA, 2009. Assessing public health capabilities during emergency preparedness tabletop exercises: reliability and validity of a measurement tool. Public Health Rep 124: 138-148.

13. Pate A, Bratberg JP, Robertson C, Smith G, 2016. Evaluation of a tabletop emergency preparedness exercise for pharmacy students. Am J Pharm Educ 80: 50.

14. Feng XY, Xia ZG, Vong S, Yang WZ, Zhou SS, 2014. Surveillance and response to drive the national malaria elimination program. Adv Parasitol 86: 81-108.

15. Wang WM, Zhou HY, Liu YB, Cao YY, Cao J, Gao Q, 2015. Establishment of malaria early warning system in Jiangsu Province IV implementation of key measures to eliminate malaria in 
Jiangsu Province in 2013 [in Chinese]. Chin J Schisto Control 27: 134-138, 161

16. Feng J, Liu J, Feng X, Zhang L, Xiao H, Xia Z, 2016. Towards malaria elimination: monitoring and evaluation of the "1-3-7" approach at the China-Myanmar border. Am J Trop Med Hyg 95: 806-810.

17. Gao $Q, 2011$. Opportunities and challenges of malaria elimination in China [in Chinese]. Chin J Schistosomiasis Control 23: 347-349.

18. Cao J, Yaobao L, Cao YY, Zhu GD, Zhou SS, 2018. Sustained challenge to malaria elimination in China: imported malaria. Chin J Parasitol Parasitic Dis 36: 4.
19. Zhang L, Feng J, Zhang SS, Jiang S, Xia ZG, Zhou SS, 2017. Malaria situation in the People's Republic of China in 2016. Chin $J$ Parasitol Parasitic Dis 35: 515-519.

20. Zhang L, Feng J, Zhang SS, Xia ZG, Zhou SS, 2018. The progress of national malaria elimination and epidemiological characteristics of malaria in China in 2017. Chin J Parasitol Parasitic Dis 36: 201-209.

21. Liu Y, Hsiang MS, Zhou H, Wang W, Cao Y, Gosling RD, Cao J, Gao Q, 2014. Malaria in overseas labourers returning to China: an analysis of imported malaria in Jiangsu Province, 2001-2011. Malar J 13: 29. 